\title{
Untangling the Concept of Task in Information Seeking and Retrieval
}

\author{
Ayah Soufan \\ ayah.soufan@strath.ac.uk \\ University of Strathclyde \\ Glasgow, UK
}

\author{
Ian Ruthven \\ ian.ruthven@strath.ac.uk \\ University of Strathclyde \\ Glasgow, UK
}

\author{
Leif Azzopardi \\ leif.azzopardi@strath.ac.uk \\ University of Strathclyde \\ Glasgow, UK
}

\begin{abstract}
Many researchers have pointed to tasks as being the driving force for Information Seeking and Retrieval (ISR). Unfortunately, researchers do not agree on what is meant by tasks and related concepts such as activities and search tasks. Researchers often use different terminologies to describe the same concepts or use the same terms to refer to different concepts. Moreover, previous researchers ignored work roles and their effect on tasks. Thus, the purpose of this paper is to present some of the main competing task hierarchies by conducting a literature analysis in the field of ISR, management, work structure, and human resources. A specific contribution is to use key existing (explicit or implicit) hierarchies and frameworks of tasks into an integrated taxonomy of tasks and related concepts for use by researchers in ISR.
\end{abstract}

\section{CCS CONCEPTS}

- Information systems $\rightarrow$ Information retrieval;

\section{KEYWORDS}

Task; Activity; Work Role; Information Seeking and Retrieval; ISR

ACM Reference Format:

Ayah Soufan, Ian Ruthven, and Leif Azzopardi. 2021. Untangling the Concept of Task in Information Seeking and Retrieval. In Proceedings of the 2021 ACM SIGIR International Conference on the Theory of Information Retrieval, Virtual Event, Canada, Fuly 11, 2021 (ICTIR '21), 9 pages.

https://doi.org/10.1145/3471158.3472259

\section{INTRODUCTION}

Tasks are one of the main driving forces for ISR and are key components that affect users' information-seeking and searching strategies. Tasks also play crucial roles in research on human behaviour [13]. However, some researchers tend to ignore the tasks being carried out in their studies. They treat tasks as a given without defining or characterising them [39]. Other researchers have considered tasks in more detail and have looked at task stages [22, 23, 38, 43], task characteristics $[5,8,43]$, or tasks performed by specific individuals in specific environments. The last one focused on academic

Permission to make digital or hard copies of all or part of this work for personal or classroom use is granted without fee provided that copies are not made or distributed for profit or commercial advantage and that copies bear this notice and the full citation on the first page. Copyrights for components of this work owned by others than the author(s) must be honored. Abstracting with credit is permitted. To copy otherwise, or republish, to post on servers or to redistribute to lists, requires prior specific permission and/or a fee. Request permissions from Permissions@acm.org.

ICTIR '21, July 11, 2021, Virtual Event, Canada

() 2021 Copyright held by the owner/author(s). Publication rights licensed to ACM. ACM ISBN 978-1-4503-8611-1/21/07 ..\$15.00

https://doi.org/10.1145/3471158.3472259 scientists [14, 17, 29, 42], students [12, 22, 32], or patent engineers [16]. ISR researchers do not, however, agree on one definition of the term 'task' and often use the same task-related terms interchangeably to describe different concepts related to tasks. Therefore, it is important to create clarity around tasks, the environment where tasks occur, and how users arrive at the point of searching for a piece of information.

In this paper, a particular contribution is to illustrate some of the key preexisting explicit and implicit task hierarchies present in other researchers' works to be able to study and compare them with each other. Additionally, we introduce concepts from management, work structure and dynamics, and human resources fields into ISR to provide an integrated task taxonomy and definitions of the concept of task and its related key-terms. Moreover, we explore the relationships among work roles, activities, work tasks, and information related tasks.

\section{BACKGROUND AND RELATED WORK}

'Task' is a generic word that can be applied to many everyday and professional activities. This paper has the focuses on tasks that incorporate an information focus, often manifesting as some kind of search activity. Researchers in the field of ISR have used the concept of 'task' in different ways and have analysed it from different perspectives. Moreover, researchers have used varying terminology to describe tasks and task attributes, and have differing accounts of the stages people go through to complete a task [7, 20, 35,43 ]. In this section, we provide an introduction to key ways in which the concept of task has been investigated in the literature, particularly looking at the stages in conducting a task, varying definitions of what constitutes a task, the characteristics of a task, and task hierarchies.

\subsection{Stages of Task}

Several researchers have examined the stages that users go through in the context of information seeking and retrieving. Kuhlthau [22] presented one of the most influential task models based on students completing an essay-based assignment. Kuhlthau described six stages of the information search process as initiation; exploration; selection; formulation; collection; and presentation. Each of these task stages are associated with distinct physical actions, cognitive thoughts, and affective feelings. Figure 1 shows an illustration of Information Search Process (ISP) stages based on Kuhlthau [22].

Building on Kuhlthau's ISP model [22], Vakkari [37] and Vakkari \& Hakala [40] identified three stages in task performance: pre-focus; formulation; and post-focus. Based on Vakkari, at the pre-focus phase, thoughts are general and actions involve seeking background information. At the formulation phase, the search for information 


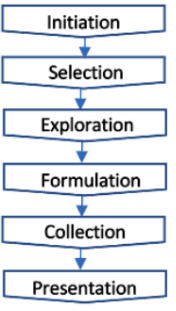

Figure 1: ISP Stages.

becomes more directed and a clearer understanding guides the individual to seek relevant information. At the post-focus phase, search becomes more specific and concentrated. Vakkari \& Hakala's study showed the effect of task performance stage on search tactics and terms choice. Additionally, Xie's [43] research validated Vakkari \& Hakala's research results. Xie characterised the same three stages as Vakkari and Vakkari \& Hakala. Figure 2 shows an illustration of task performance stages based on Vakkari [37], Vakkari \& Hakala[40] and Xie [43].

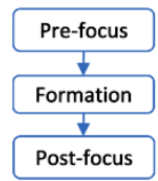

Figure 2: Task Performance Stages.

Byström \& Hansen [7] divided task performance into three main parts: construction; actual performance; and completion. The construction part consists of comprehending the preconditions and goals for performance and completion in relation to a given assignment. The actual performance part consists of the practical and conceptual actions taken to achieve the goals. The completion part includes evaluating task resolution to modify or accept it as a final task resolution. Figure 3 shows an illustration of task performance parts based on Byström \& Hansen [7].

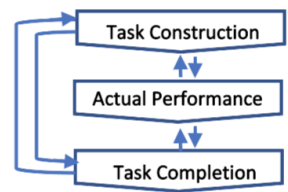

Figure 3: Task Performance Parts

Järvelin et al. [20] focused on information interactions. For Järvelin et al., information interactions are the behavioural and cognitive activities related to task planning; searching information items; selecting between information items; working with information items; and synthesising and reporting. Based on Järvelin et al. these five activities are essential in learning tasks and contribute to task performance and outcome. According to Järvelin et al., information interaction is broader than searching and subsumes information access; task-based searching; or task-based information retrieval. Figure 4 shows an illustration of information interaction activities based on Järvelin et al. [20].

The reviewed literature shows that different researchers characterised different stages that individuals go through while working on their tasks. Researchers used different terms but their concepts

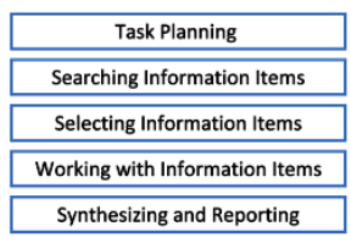

Figure 4: Information Interaction Activities.

are the same. For example, Vakkari [37], Vakkari \& Hakala [40], and Xie [43] identified three stages of tasks, unlike Kuhlthau [22] who identified six. Vakkari's pre-focus stage corresponds to Kuhlthau's 'initiation' and 'selection' stages. Vakkari's post-focus stage associates with Kuhlthau's 'collection' and 'presentation' stages. In addition, among Byström \& Hansen's [7] three main parts of task performance, the construction part can be divided into the 'initiation', 'selection', 'exploration' and 'formulation' of Kuhlthau's ISP model. Kuhlthau's ISP model was initially developed in connection to a school assignment task, but Byström \& Hansen focused on tasks in work settings which is why they did not put much emphasis on the construction phase as Kuhlthau did. Byström \& Hansen believed that people are more confident in their judgments in performing their everyday work tasks and are less uncertain of the requirements of the tasks. The 'actual performance' and 'task completion' parts developed by Byström \& Hansen can be mapped to the 'collection' and 'presentation' stages of Kuhlthau's ISP model. Figure 5 shows an illustration of tasks stages based on Byström \& Hansen [7], Kuhlthau [22], and Vakkari \& Hakala [40].

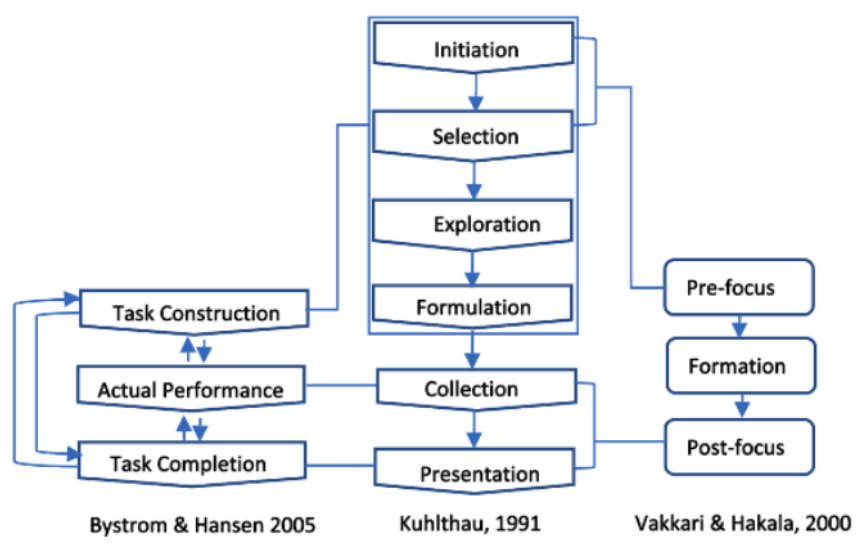

Figure 5: Task Stages.

\subsection{Tasks and Sub-Tasks}

In the ISR literature, tasks are sometimes called work tasks, information seeking tasks, search tasks, or information retrieval tasks. Some researchers restrict the term 'task' to work tasks that might trigger information search tasks [39]. Other researchers view work tasks as a motivation of information-seeking and information search tasks $[15,27]$.

Järvelin et al. [20] understands tasks as the larger tasks motivating information interaction. Byström \& Hansen [7] define three levels of tasks including work tasks; information-seeking tasks; and information search tasks. According to Byström \& Hansen, work tasks are to some degree outlined by the work organisation 


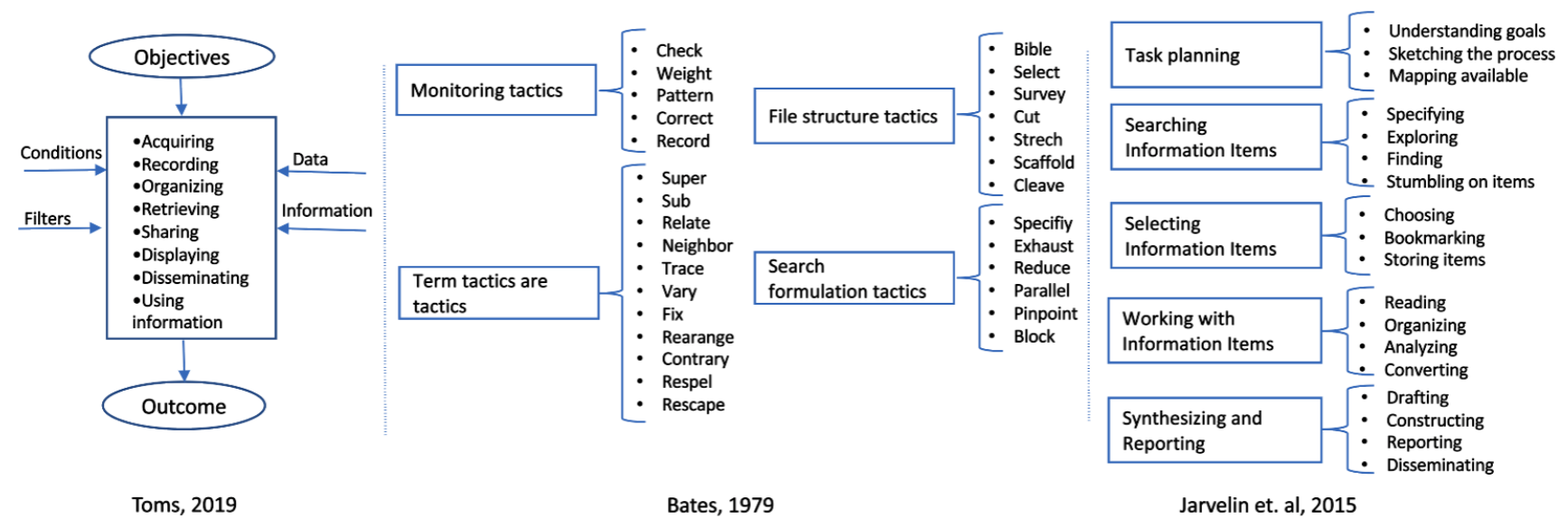

Figure 6: Information Processes, Tactics and Information Activities.

which provides the environment or domain to which a task belongs. Vakakri [39] defines a task as an activity to be performed to accomplish a goal. Xie [43] classifies tasks into two levels: work and search tasks. While a work task leads to information searching, a search task determines what an individual is searching for [43] For Xie [42], tasks and goals are inseparable in the ISP process Xie [42] describes goal structure to represent four levels of goals including long-term goal; leading search goal; current search goal; and interactive intentions. For Toms [35], a work task is explicit and has a goal to be achieved through following a set of instructions. Toms [35], Järvelin et al. [20], Xie [43], Byström \& Hansen [7] and Vakakri [39] believe that a task has a recognisable beginning and end. Additionally, a task may range from a simple one requiring little thought, to a very complex decision-making task. Complex tasks consist of smaller sub-tasks and both a large task or any of its sub-tasks may be considered as a task as well. Those sub-tasks must be accomplished and connected to reach a meaningful result.

\subsection{Search Tasks, Information Processes and Tactics}

Several researchers have examined information related tasks, particularly search tasks and search actions. Based on Byström \& Hansen [7], information search tasks are sub-tasks to an information-seeking task, and information-seeking tasks are sub-tasks of a work task. For Byström \& Hansen, information-seeking tasks focus on the satisfaction of an entire information need through consultations of several channels and sources. While information search tasks focus on the satisfaction of a fraction of an information need through searching for information from one or more sources.

Based on Toms [35], a task/sub-task may use one or more information processes and actions. Toms defines a process as a set of partially ordered steps intended to reach a goal. Thus, information processes are those actions and operations that modify or augment information so that the original unit of data or information changes in some fashion. For Bates [3], a move is the basic unit of analysis, and the identifiable thought or action of information searching behaviour. In addition, based on Bates, a tactic is a move made to further a search. Tactics are primarily designed to help in more complex searches that involve many stages. Bates defines twentynine tactics used in information searching which are grouped into four categories: monitoring; file structure; search formulation; and term tactics. While a tactic deals with short-term goals, a strategy deals with overall planning in the context of information searching.

Järvelin et al. [20] identify generic activity types that are performed across the task process stages related to information interaction. Järvelin et al. understand information interaction as behavioural and cognitive activities related to task planning, searching and selecting information items, working with information items, and synthesising and reporting. Figure 6 shows an illustration of information processes, tactics and activities based on Toms [35], Bates [3], and Järvelin et al. [20].

The reviewed literature shows that different researchers used different terms to describe or categorise information-related tasks, which are related to actions such as searching, acquiring, organising, synthesising, disseminating, and using information.

\subsection{Tasks Characteristics}

In addition to describing the nature of a task, task characteristics or what makes tasks different have been discussed in different ways in the ISR field [7, 9, 15, 43]. Li \& Belkin [26] did great work in identifying some of the essential task facets and attributes. Li \& Belkin categorised those facets of work tasks and information search tasks into generic attributes including source of task; task doer; time; action; product; and goal, and common attributes including task characteristics and user's perception of task. The following subsections describe some of the main task characteristics that have been studied in the ISR literature:

2.4.1 Task Complexity. Task complexity is one of the most important and most studied attributes of tasks in ISR. Task complexity affects the performance and the information needed to complete a task [36]. For Campbell [9], task complexity is related to the following characteristics of a task: repetitivity; analysability; the number of alternative paths of task performance; and outcomes novelty. Campbell characterises tasks based on their complexity into five categories: simple tasks; decision tasks; judgment tasks; problem tasks; and fuzzy tasks. 
Task complexity can be classified as objective and subjective. Based on Adewale [2], subjective task complexity is studied from the task performer's point of view, and objective task complexity has to do with the feature of the task itself, not the performer. Campbell [9] concludes that any objective task characteristic that implies an increase in information load, information diversity, or rate of information change can be considered a contributor to complexity.

Byström \& Järvelin [8] divide tasks based on the predeterminability of information requirements, process, and output of the task into five categories: automatic information processing tasks; normal information-processing tasks; normal decision tasks; in-known tasks; and genuine decision tasks. Likewise, Byström [6] classifies tasks according to their complexity into three classes: automatic information processing tasks; normal information processing tasks; and decision tasks. Byström \& Järvelin conclude that when the task complexity increases, the complexity of information needed and the number of sources increase as well. According to Kuhlthau [22] and Vakkari [36], task complexity is associated with the degree of uncertainty of task performance.

2.4.2 Origin of Tasks. Xie [43] classifies tasks based on the origination of the task into two types: self-generated and assigned tasks. Self-generated tasks refer to the tasks that participants came up with themselves. Assigned tasks refer to tasks that are delegated or suggested by people other than the participants themselves. Byström \& Hansen [7] classify tasks based on their origin into: subjective and objective tasks. Subjective tasks are seen as internal to the performer and defined by themselves. Objective tasks are external to the performer and imposed on them. These types of origination of tasks aligned with Hackman's [13] task classification.

2.4.3 Structured $\mid$ Unstructured Tasks. Based on Hansen [15], structured tasks have a designed course, whereas unstructured tasks may involve creative planning and flexibility. For Toms [35], structured tasks are mostly instructional, and they leave little discretion to the worker. Meanwhile, unstructured tasks are constraints-based, epitomize cognitive work, and require significant mental effort in combination with knowledge and skills.

2.4.4 Nature of Tasks. Xie [43] identifies three types of tasks: routine; typical; and unusual. Based on Xie's study, routine tasks are repetitive tasks. Typical tasks are new tasks to the performer but they are similar to tasks which they used to perform. Unusual tasks are new tasks to the performer and they are not similar to tasks that they used to perform.

2.4.5 TimeFrame. According to the time needed to fulfill tasks, Xie [43] classifies the timeframe into extremely urgent; urgent; and non-urgent. For Xie, extremely urgent means that the task has to be accomplished within half an hour. Urgent means that the task has to be accomplished within twenty-four hours. Non-urgent means that the task can be accomplished in more than twenty-four hours.

The above characteristics are some of the key characteristics tasks, which are just one level of the task hierarchy.

\subsection{Task Hierarchies}

Several researchers proposed various task hierarchies and structures. Some of these hierarchies were explicitly mentioned in the previous papers such as Toms' task hierarchy [35]. In most cases, these were implicit such as in the works of Byström [7], Xie [43], and Järvelin et al. [20]. We have constructed the different task hierarchies and structures based on our readings in the mentioned works.

We believe that these hierarchies and structures are helpful and have several strengths as well as weaknesses. Thus, we used the most significant hierarchies and unified them to retain the strengths and expand them with more concepts from the work environment to propose an integrated task taxonomy that we believe can be used within various work environments and real-life contexts.

Toms [35] built a task hierarchy that describes work tasks and shows the relationships between activities, tasks, and information processes. For Toms, work is made of a set of activities and tasks. Activities are defined within the scope of a worker's job description, and they provide the goals for individual work roles. Toms put activities at the highest level of the task hierarchy. Activities may consist of one or more tasks. For Toms, a task is explicit and has a goal to be achieved through following a set of instructions. In addition, a work task consists of one or more sub-tasks, and the task itself may be considered as a sub-task to a larger project. Those sub-tasks must be accomplished and connected in order to reach a meaningful result. Information processes are in the lower level of Toms' task hierarchy. Based on Toms, information processes are those actions and operations that modify or augment information so that the original unit of data or information changes in some fashion.

According to Xie [43], task and goal are inseparable in the information seeking and retrieving process. Based on Xie, there are four levels of goal structures (hierarchy): long-term goal, leading search goal, current search goal, and interactive intentions. For Järvelin et al. [20], a task is a sequence of activities a person performs to accomplish a goal. Based on Byström \& Hansen [7], work task is at the top level, information seeking task are sub-tasks to a work task, and information search tasks are sub-tasks to an information seeking task. Figure 7 shows an illustration of the different task hierarchies based on Toms [35], Xie [43], Byström \& Hansen [7] and Järvelin et al. [20].

According to Taylor [34], "tasks do not exist independently but in interaction with the context of which they are a part". Various researchers talked about or corporated the socio-organisational context in their ISR models or hierarchies [4, 28], but their contributions had issues with simulating the real-life experience in work environments. Their models fell short of describing work roles or their effect on information seeking and retrieval tasks. For Toms [35], there is no clear difference between a job description or a job role; both can define the core activities. Other hierarchies and task models ignored activities and work roles completely. We believe that the previous hierarchies and structures are important but they are not complete and do not take into consideration the real-life scenarios at workplaces. 


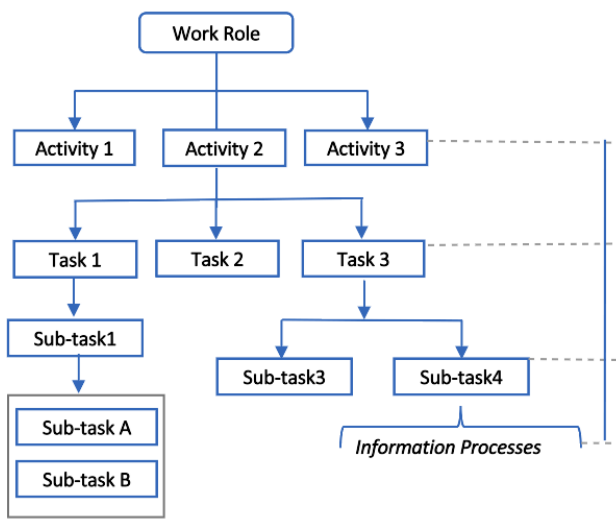

Toms 2019

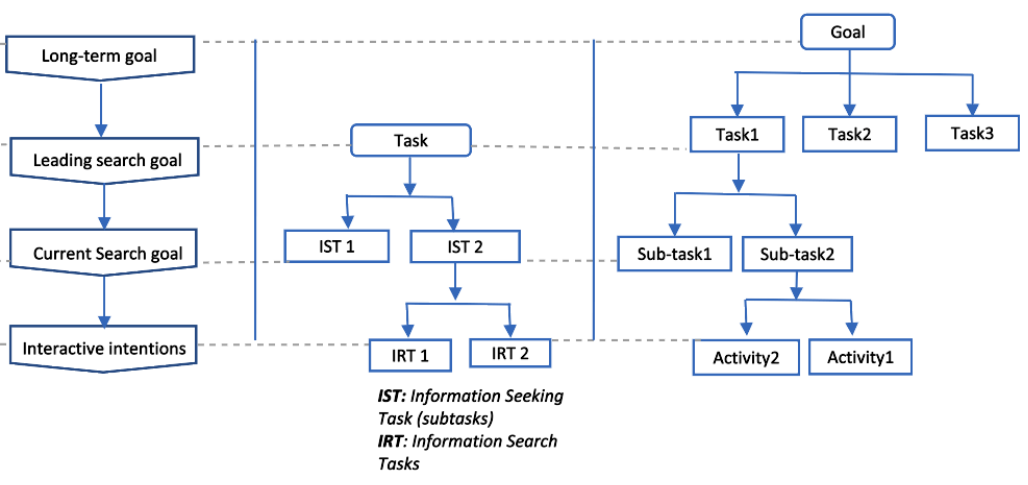

Bystrom \& Hansen 2005

Jarvelin and others 2015

Figure 7: Task Hierarchies.

To summarise, previous research has studied the concepts of tasks from different perspectives. It has studied stages of tasks, levels of tasks, nature of tasks, the complexity of tasks, and the origin of tasks. Ultimately, previous research did not agree on the same definitions for tasks and the related key terms. The same terms are often used interchangeably to describe different tasks concepts. Additionally, the proposed various task hierarchies (explicit or implicit) do not agree on the components of task hierarchies and the involvement of work roles and their effect on tasks. Therefore, we propose an integrated task taxonomy, which we believe draws a full picture of tasks and their relationships with work roles, activities, sub-tasks and information related tasks in work environments. Our task taxonomy can also be used in real-life contexts as well as work environments. Moreover, we provide a series of working definitions that will help in solving the inconsistency in the literature related to tasks and their related key terms.

\section{METHODOLOGY}

We reviewed studies related to task-based searching, information tasks, tasks complexity, tasks conceptual models, and general models of information-seeking of professionals. To understand more about work roles in the work environments and how they might affect information-related tasks, we reviewed literature related to role theory, management, work structure and dynamics, and human resources. We focused on peer-reviewed papers published at some of the top conferences and journals such as the Academy of Management Journal, Information Processing and Management, Journal of the American Society for Information Science and Technology, The Annual Review of Information Science and Technology, and Conceptions of Library and Information Sciences conference. We were interested in high-quality and highly cited papers that studied and defined tasks, their different types, their levels, stages, and their key attributes. We were also interested in papers that combined management and work structure concepts with ISR. We did forward and backward chaining to find more relevant papers and studies in the field.

We grouped the reviewed papers based on their content. We studied each group and worked on finding the key similarities and differences between them. We noticed that some papers were describing the same task-related concepts using different terms. However, sometimes the same term was used to describe different concepts. We were able to find common themes and connections between the different papers and studies. Moreover, we noticed that work roles were rarely mentioned in the ISR field. Therefore, we studied the papers that combined management and work structure concepts with ISR. We also studied papers related to management, human resources, and work structure and dynamics to gain a better understanding of tasks and work roles in the work environments.

In sum, we worked on three approaches: 1) we studied and compared the various papers to define and map the same concepts that were mentioned using different terms across them; 2) we studied papers from management and human resources fields to have a better understanding of work tasks and work roles concepts; and 3) we used the previous task hierarchies to inform our taxonomy and include some of the important missing concepts.

In the next section, we introduce our integrated task taxonomy as well as two real-life examples of the taxonomy.

\section{INTEGRATED TASK TAXONOMY}

ISR researchers who studied work tasks ignored the fact that individuals may take a limitless number of potential roles at work [11]. The literature in work structure, management, and human resources shows that information need is highly related to the performance of a particular role and its associated activities and tasks. Thus, we propose a task taxonomy that builds on the previous task hierarchies and completes them. Also, we provide a series of working definitions of work role, activity, and task $\$ sub-task.

\subsection{Work Roles}

Most researchers in the field of information seeking and retrieval ignored the different roles taken by individuals while working on their tasks even though work roles have an effect on information-related tasks. The reviewed literature suggests that the same task might be approached in different ways depending on the performer's role at the time of completing the task. Some researchers implicitly 


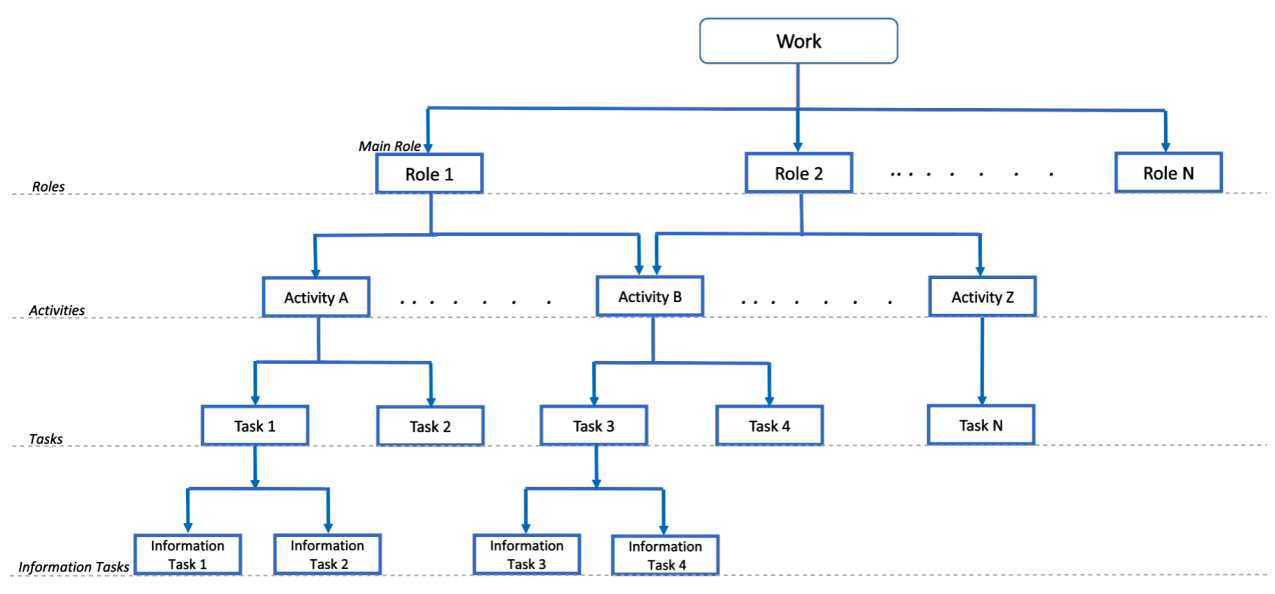

Figure 8: Integrated Task Taxonomy.

mention work roles and their effect on the work and informationrelated tasks. For example, Järvelin \& Wilson [21] raise a crucial point about work roles and the importance of thinking of tasks from a context or discipline point of view. Järvelin \& Wilson make it clear that there may be significant differences in the nature of the tasks from one department to another in the same administration. Based on Järvelin \& Wilson, different departments may have different concerns which can lead to approach the same tasks in different ways. Thus, it is important to study the context of tasks and work roles taken by individuals to complete their tasks. Leckie et al. [24] reviewed research related to the information-seeking studies for engineers, health care professionals, and lawyers. Leckie et al. found that it is common that professionals play many distinct roles throughout any given day. Besides, Leckie's et al. review concludes that different tasks are associated with different roles taken by the same employee, which generate different information needs that must be met to move forward with work. Leckie's et al. study shows that professionals who work on complex responsibilities are expected to take different technical, managerial, and interpersonal roles. Sonnenwald [33] studied the boundary-spanning roles in the design process for four engineering-design situations. Sonnenwald's study shows that individuals may assume one or more roles and may change roles during the design process.

The literature in the field of management and human resources assures that individuals can take multiple work roles while working on their tasks and duties inside their organisations [10, 11, 41]. Based on Huvila [18], an individual in a work environment may be required to work on a set of activities and tasks to accomplish a goal. Besides, most work environments are dynamic. Thus, individuals may perform on multiple roles at the same time [18]. Even though job description specifies details about the job responsibilities, requirements, skills, knowledge, and expertise which are needed to fill a certain work role, employees may have multiple roles that may be extended to other different sub-roles [11].

Work roles can not be divorced from the organisational context within which they exist [19]. Even though some organisations are more hierarchical than others, employees in flat organisations also have roles and tasks. Parker et al. [31] show that employees do not react passively to the jobs, tasks, roles, and goals assigned by their managers. Rather, they actively change, shape or expand their work roles. Griffin et al. [11] suggest that work roles must emerge dynamically in response to changing conditions and demands in work environment. They believe that role behaviours can contribute to effectiveness at three levels including individual, team, and organisation.

Roles refer to the social position people have and the behaviour associated with that position (e.g., mother, teacher, and lawyer) [1]. Work roles are "behaviours and activities that are directly associated with achieving specific objectives" [19]. Employees may have a work role that matches their domain of knowledge and expertise, which can be called the main role, but they have to perform other roles within the organisation environment to fulfill all their duties and responsibilities [24]. Besides, some roles can be extended to other different sub-roles [19, 24]. Moreover, an employee can perform in multiple work roles at the same time or share the same work roles with others [18].

Here is a summary of the main characteristics of a work role:

- Work roles exist within an organisational context [19].

- A work role can be extended to other sub-roles [19, 24].

- There are two types of roles: main and secondary roles [24].

- Individuals can perform multiple work roles at the same time [18].

- More than one individual can perform the same role [18].

- A work role can define a series of core activities [35].

\subsection{Activity}

In the literature of ISR, some researchers defined and used the term 'activity' in their research, others did not. While Toms [35] believes that an activity may consist of a set of tasks, some researchers believe that the opposite is true. For example, Järvelin et al. [20] define the task as a sequence of activities a person performs to accomplish a goal. Also, Li \& Belkin [27] view work task as an activity people perform to fulfill their responsibility for their work. According to Toms, work is made of a set of activities and tasks. Activities provide the goals for individual work roles. Toms adopts Norman's [30] task hierarchies in defining work activities. Norman's hierarchical structure comes from his Activity Theory. According to Norman, activities are not the same as tasks. An activity is a coordinated and integrated set of tasks. Based on Toms, activities are defined 


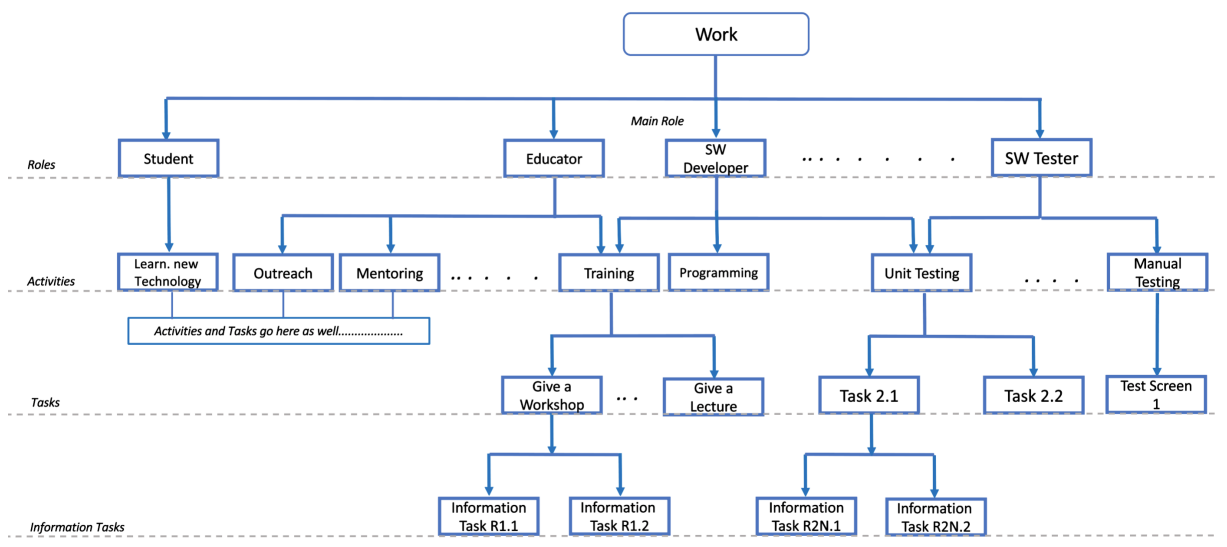

Figure 9: Task Hierarchy for a Software Developer.

within the scope of a worker's job description, and they are implicit in discussions of work. Toms puts activities at the highest level of the task hierarchy. She believes that activities may consist of one or more tasks. Meanwhile, Xie [43] believes that tasks and goals are inseparable in the information seeking and retrieving process. Xie does not mention activities in her goal task structure. Xie's 'Long-term goal', which resides at the top of her goalltask structure refers to a user's personal goal that they pursue for a long time such as professional achievement. Such goal is similar to Toms' activity concept. Activities can be implicit or explicit depending on the activities themselves and the individuals who work on them. For example, Software Engineers who work in tech companies need to keep learning about better ways to build their products. 'Learning' activities can be implicit in this case. However, other activities such as supervising other engineers and attending conferences can be explicit activities.

In summary, an activity is an abstract concept that contains one or more tasks that might expand and generate more tasks depending on the task and the one who performs them. An activity has a goal and objective within the organisational environment. Activity is long-running and open-ended, which does not have a recognisable beginning and end. An activity is flexible and may be semi-structured. Here are some of the main characteristics of activities:

- Activities exist within an organisational context [35].

- Activities are generic and may not have specific guidelines to follow [35].

- An activity may consist of one or more tasks [35].

- An activity may be associated with one or more work roles.

- An activity may consist of a set of sub-activities.

- Activities can be implicit [35] or explicit.

- Activities have goals and objectives.

- Activities are open-ended.

- Activities are long-running.

\subsection{Tasks}

Work task are often seen as the motivation for information-seeking and information search tasks [25]. Järvelin et al. [20] understand tasks as the larger tasks motivating information interaction. Byström
\& Hansen [7] define three levels of tasks including work tasks; information-seeking tasks; and information search tasks. Xie [43] classifies tasks into two levels: work and search tasks, and her goal task structure describes four levels of work tasks. For Toms [35], an activity consists of one or more tasks, and a work task is explicit and has a goal to be achieved through following a set of instructions. Additionally, tasks have recognisable beginnings and ends despite not being recognisable the moment they are happening [7]. Tasks can be accomplished by following some guidelines, and can also be measured. A task may range from almost automatic processing requiring little thought, to very complex decision-making $[7,20,35]$. Complex tasks consist of smaller sub-tasks. Both a large task or any of its sub-tasks may be considered as a task [21].

In summary, tasks are what someone does to achieve an activity's goal under a specific work role. They can be given to or identified by the individuals themselves. Tasks have recognisable beginnings and ends. Besides, tasks have goals and objectives within the organisational environment and they can be measured. Moreover, tasks have constraints, requirements to fulfill, and they may range from simple to complex decision-making. Tasks can be divided into smaller sub-tasks. Here are some of the main characteristics of tasks:

- Tasks are either assigned or self generated [7, 8, 13, 43].

- Tasks have recognisable beginnings and ends [8, 20, 35, 39].

- A task has a practical goal (some form of output) [7, 8, 20,35].

- A task has a meaningful purpose (a reason)[7].

- A task has requirements to fulfill $[7,35]$.

- A consequence of the last point is that a task's progress can be measured to some degree.

- A task may range from simple to very complex decisionmaking[7, 8, 20, 35].

- A task can be structured or unstructured $[15,35]$.

- A task may have some constraints[35].

- A task can be divided into smaller sub-tasks [7, 8, 20, 35, 43].

- Each sub-task has a goal.

- Sub-tasks may need to be undertaken together to build a meaningful whole.

- Sub-tasks may be further decomposed into more sub-tasks.

- Sub-tasks may consist of or contain information seeking and retrieval tasks. 


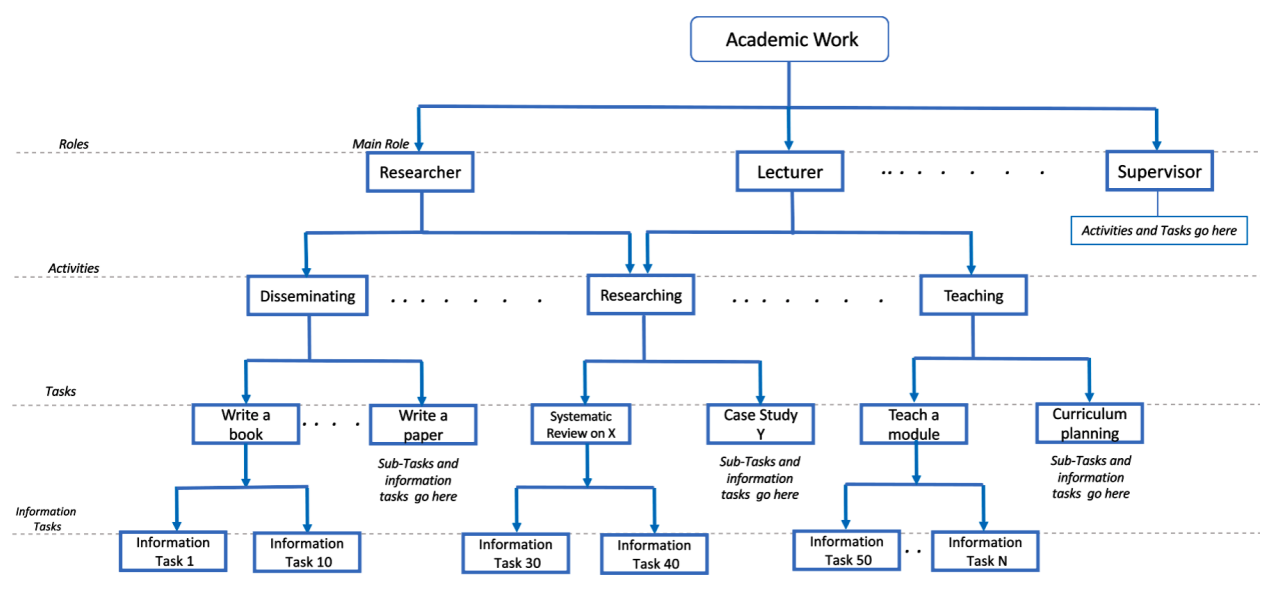

Figure 10: Task Hierarchy for an Academic.

Figure 8 shows the integrated taxonomy and the relationships between work roles, activities, tasks and information related tasks.

Information-related tasks are at the lowest level of the task taxonomy. As the name suggests, those tasks deal with information. Our aim in this paper is to provide a general taxonomy that shows the relationship between work roles, activities, tasks, and informationrelated tasks. Information-related tasks might need more extensive studying and analysis as they include many details regarding information resources, information systems, and human behaviour on using the different information sources and systems. Therefore, the analysis of information-related tasks is beyond the scope of this paper.

\subsection{Real Life Examples}

In this section we illustrate the integrated task taxonomy using two examples of how the taxonomy can be applied to working environments.

4.4.1 Software Engineer Example. A Software Engineer in one of the tech companies may take several work roles to fulfill their responsibilities and job duties such as being a software developer, software tester, educator, and student. The core activities for a developer might include programming, testing, and training activities. Each one of these activities consists of one or more tasks. Programming activity may consist of tasks such as maintenance, bug-fixes, or programming a new feature for a specific project. Programming a new feature in a specific project may include several sub-tasks such as building a specific screen or programming a new button that provides a specific service. These tasks may require a series of information-related tasks. For a Software engineer, building a specific screen to teach or train junior programmers may be different than building the screen for a specific project that provides a service used by millions of users. In the first case, the software engineer|trainer may be more interested in simplifying the concept for the trainees to understand, so when they search for a piece of code, they are interested in finding a well-written and simple code. But in the second case, the software engineer|programmer is interested in shipping a bug-free code that is reliable and scalable, so their focus is on a high-quality code that provides the needed scalability, even though the code itself might be so complicated. In sum, different work roles result in different information needs and different behaviours in seeking information. Figure 9 shows the work hierarchy and the relationships between work roles, activities, tasks, and information related tasks for a software engineer.

4.4.2 An Academic Example. An academic in a university may take several roles such as being a researcher, head of a school, lecturer, manager, or supervisor. Each role of these may consist of a series of activities. For example, a researcher may work on disseminating or researching activities. Disseminating activity may consist of several tasks such as writing a book or a conference paper. On the other hand, a lecturer may work on researching and teaching activities. Researching activity may be associated with two roles: researcher and lecturer. An academic may work with their students on a research project as part of a taught module or as a researcher who conducts research as part of their responsibilities and requirements at the institute. The research activity may consist of several sub-activities and tasks such as working on a specific case study and completing a literature review on a specific topic, etc. Being a lecturer includes teaching specific modules or planning a specific curriculum. A task of teaching a module may consist of a series of tasks including preparing slides, prepare a reading list, marking, etc. Each one of these tasks may consist of one or more information-related tasks. Figure 10 shows the work hierarchy and the relationships between work roles, activities, tasks and information-related tasks for an academic.

\section{CONCLUSION}

The previous section presented a task taxonomy that provides working definitions of work roles, activities, and tasks. It also provides normalisation of the different tasks key-terms. Additionally, the presented taxonomy shows the relation of work roles, activities, tasks, sub-tasks, and information-related tasks in an organizational context. Such taxonomy is clearly needed in clarifying the inconsistency in the literature regarding tasks.

Moreover, our integrated task taxonomy can help ISR researchers better understand tasks; different levels of tasks; the effect of work roles on activities, task, and information-related tasks. Consequently, this will help them better design tasks for their different studies and 
experiments. That will help in building better information systems which are able to offer different types of support for the different work roles and levels of tasks.

The proposed taxonomy draws a clearer picture that allows for a better understanding of the environment where tasks occur, and how individuals arrive to the point of searching for a piece of information. Furthermore, this taxonomy can be applied to real-life as well as organisational contexts.

The integrated task taxonomy is one step toward building up the knowledge base in the ISR field. But there is room for further work to understand the details of the relation between the different levels in the hierarchy. Work roles are composed of a series of activities, but the question is whether a given set of activities define the role or if the role define the set of activities. Is the relation between roles and activities bottom-up, top-down, or both? Further work and analysis need to be done to answer these questions.

\section{ACKNOWLEDGEMENT}

We would like to thank Professor Elaine Toms for her valuable comments on this research work and the reviewers for their helpful suggestions and feedback. The work reported here is funded by the DOSSIER project under European Union's Horizon 2020 research and innovation program, Marie Skłodowska-Curie grant agreement No 860721

\section{REFERENCES}

[1] Marja Aartsen and Thomas Hansen. 2020. Social Participation in the Second Half of Life. (2020).

[2] Nafisat Toyin Adewale, Yushiana Mansor, Muhammad-Bashir Owolabi Yusuf, and Ahmeed Onikosi. 2017. Uncertainty and subjective task complexity in the information-seeking behaviour of lawyers. Library Review (2017).

[3] Marcia J Bates. 1979. Information search tactics. Fournal of the American Society for information Science 30, 4 (1979), 205-214.

[4] David Bawden. 2007. The Turn: Integration of Information Seeking and Information Retrieval in Context. Fournal of Documentation (2007).

[5] Katriina Byström. 1999. Task complexity, information types and information sources: examination of relationships. Tampere University Press.

[6] Katriina BystrÖm. 2002. Information and information sources in tasks of varying complexity. Journal of the American Society for information Science and Technology 53, 7 (2002), 581-591.

[7] Katriina Byström and Preben Hansen. 2005. Conceptual framework for tasks in information studies. Fournal of the American Society for Information science and Technology 56, 10 (2005), 1050-1061.

[8] Katriina Byström and Kalervo Järvelin. 1995. Task complexity affects information seeking and use. Information processing \& management 31, 2 (1995), 191-213.

[9] Donald J Campbell. 1988. Task complexity: A review and analysis. Academy of management review 13, 1 (1988), 40-52.

[10] Adam M Grant and Susan J Ashford. 2008. The dynamics of proactivity at work. Research in organizational behavior 28 (2008), 3-34.

[11] Mark A Griffin, Andrew Neal, and Sharon K Parker. 2007. A new model of work role performance: Positive behavior in uncertain and interdependent contexts. Academy of management journal 50, 2 (2007), 327-347.

[12] Jillian R Griffiths and Peter Brophy. 2005. Student searching behavior and the web: use of academic resources and Google. (2005)

[13] J Richard Hackman. 1969. Toward understanding the role of tasks in behavioral research. Acta psychologica 31 (1969), 97-128.

[14] Lotta Haglund and Per Olsson. 2008. The impact on university libraries of changes in information behavior among academic researchers: a multiple case study. The journal of academic librarianship 34, 1 (2008), 52-59.

[15] P Hansen. 1999. User interface design for IR interaction. a task-oriented approach. Third International Conference on the Conceptions of the Library and Information Science (1999), 191-205.

[16] Preben Hansen and Kalervo Järvelin. 2000. The information seeking and retrieval process at the Swedish Patent and Registration Office. In Proc. ACM SIGIR Workshop on Patent Retrieval.

[17] Bradley M Hemminger, Dihui Lu, KTL Vaughan, and Stephanie J Adams. 2007. Information seeking behavior of academic scientists. Fournal of the American society for information science and technology 58, 14 (2007), 2205-2225.
[18] Isto Huvila. 2008. Work and work roles: a context of tasks. fournal of documentation (2008)

[19] Daniel R Ilgen and John R Hollenbeck. 1991. The structure of work: Job design and roles. (1991)

[20] Kalervo Järvelin, Pertti Vakkari, Paavo Arvola, Feza Baskaya, Anni Järvelin, Jaana Kekäläinen, Heikki Keskustalo, Sanna Kumpulainen, Miamaria Saastamoinen, Reijo Savolainen, et al. 2015. Task-based information interaction evaluation: The viewpoint of program theory. ACM Transactions on Information Systems (TOIS) 33, 1 (2015), 1-30.

[21] Kalervo Järvelin and Thomas D Wilson. 2003. On conceptual models for information seeking and retrieval research. Information research 9, 1 (2003), 9-1.

[22] Carol C Kuhlthau. 1991. Inside the search process: Information seeking from the user's perspective. Fournal of the American society for information science 42, 5 (1991), 361-371.

[23] Carol C Kuhlthau, Jannica Heinström, and Ross J Todd. 2008. The 'information search process' revisited: Is the model still useful. Information research 13, 4 (2008), 13-4

[24] Gloria J Leckie, Karen E Pettigrew, and Christian Sylvain. 1996. Modeling the information seeking of professionals: A general model derived from research on engineers, health care professionals, and lawyers. The Library Quarterly 66, 2 (1996), 161-193.

[25] Yuelin Li. 2009. Exploring the relationships between work task and search task in information search. Fournal of the American Society for information Science and Technology 60, 2 (2009), 275-291.

[26] Yuelin Li and Nicholas J Belkin. 2008. A faceted approach to conceptualizing tasks in information seeking. Information Processing \& Management 44, 6 (2008), 1822-1837.

[27] Yuelin Li and Nicholas J Belkin. 2010. An exploration of the relationships between work task and interactive information search behavior. Fournal of the American Society for information Science and Technology 61, 9 (2010), 1771-1789.

[28] Elisabeth Logan. 1993. Information retrieval interaction: P. Ingwersen. Taylor Graham, Los Angeles (1992). x+ 246 pp., \$55, ISBN 0-947568-54-9. (1993).

[29] Xi Niu, Bradley M Hemminger, Cory Lown, Stephanie Adams, Cecelia Brown, Allison Level, Merinda McLure, Audrey Powers, Michele R Tennant, and Tara Cataldo. 2010. National study of information seeking behavior of academic researchers in the United States. Fournal of the American Society for Information Science and Technology 61, 5 (2010), 869-890.

[30] Donald A Norman. 2005. Human-centered design considered harmful. interactions 12, 4 (2005), 14-19.

[31] Sharon K Parker, Toby D Wall, and Paul R Jackson. 1997. "That's not my job”: Developing flexible employee work orientations. Academy of management journal 40, 4 (1997), 899-929.

[32] Mikko Pennanen and Pertti Vakkari. 2003. Students' conceptual structure, search process, and outcome while preparing a research proposal: a longitudinal case study. Fournal of the American Society for Information Science and Technology 54, 8 (2003), 759-770.

[33] Diane H Sonnenwald. 1996. Communication roles that support collaboration during the design process. Design studies 17, 3 (1996), 277-301.

[34] R. Taylor. 1991. Information use environments. Progress in communication sciences (1991), 217-255.

[35] Elaine G Toms. 2019. Information activities and tasks. Information at Work: Information Management in the Workplace (2019), 33.

[36] Pertti Vakkari. 1999. Task complexity, problem structure and information actions: Integrating studies on information seeking and retrieval. Information processing \& management 35, 6 (1999), 819-837.

[37] Pertti Vakkari. 2000. Relevance and contributing information types of searched documents in task performance. In Proceedings of the 23rd annual international ACM SIGIR conference on Research and development in information retrieval. 2-9.

[38] Pertti Vakkari. 2001. A theory of the task-based information retrieval process: A summary and generalisation of a longitudinal study. Fournal of documentation (2001).

[39] Pertti Vakkari. 2003. Task-based information searching. Annual review of information science and technology 37, 1 (2003), 413-464.

[40] Pertti Vakkari and Nanna Hakala. 2000. Changes in relevance criteria and problem stages in task performance. Fournal of documentation (2000).

[41] Theresa M Welbourne, Diane E Johnson, and Amir Erez. 1998. The role-based performance scale: Validity analysis of a theory-based measure. Academy of management journal 41, 5 (1998), 540-555.

[42] Hong Xie. 2000. Shifts of interactive intentions and information-seeking strategies in interactive information retrieval. Journal of the American Society for information Science 51, 9 (2000), 841-857.

[43] Iris Xie. 2009. Dimensions of tasks: influences on information-seeking and retrieving process. Fournal of Documentation (2009). 